\title{
Continuity and Invariance of Hybrid Automata
}

\author{
John Lygeros ${ }^{1}$, Karl Henrik Johansson² ${ }^{2}$ Slobodan N. Simić ${ }^{3}$, Jun Zhang, Shankar Sastry
}

\begin{abstract}
Hybrid automata have been proposed as a language for modelling and analysing the interaction of digital and analogue dynamics in embedded computation systems. In this paper, hybrid automata are studied from a dynamical systems perspective. Extending earlier work on conditions for local existence and uniqueness of executions of hybrid automata, we characterise a class of hybrid automata whose executions depend continuously on the initial state. The continuity conditions are subsequently used to derive an extension of LaSalle's principle for studying the stability of invariant sets of states of hybrid automata.
\end{abstract}

Keywords: Hybrid systems; Dynamical systems; Continuity; LaSalle's Invariance Principle.

\section{Introduction}

Despite intense research activity in recent years, many fundamental questions regarding the dynamical properties of hybrid systems still remain unresolved. In this paper, we try to address such problems for a fairly large class of hybrid systems, known as hybrid automata. This class is rich enough to subsume a number of interesting subclasses such as switched systems [1], complementarity systems [2], and piecewise linear systems [3]. Earlier work by the authors [4] centred around conditions for existence and uniqueness of executions for hybrid automata. Global existence was also studied, in the context of Zeno executions, i.e. executions that take an infinite number of discrete transitions in a finite amount of time [5]. The results were subsequently used to study problems in behavioural robotics [6], and extend Lyapunov's linearisation method to classes hybrid automata [7].

In this paper, this line of work is pursued further by establishing conditions that guarantee that the execu-

\footnotetext{
${ }^{1} \mathrm{~J}$. Lygeros is with the Department of Engineering, University of Cambridge, Cambridge CB2 1PZ, U.K. Email: j1290@eng.cam.ac.uk

${ }^{2}$ K. H. Johansson is with the Department of Signals, Sensors \& Systems, Royal Institute of Technology, 10044 Stockholm, Sweden. Email: kallej@s3.kth.se.

${ }^{3}$ S. N. Simić, Jun Zhang, and Shankar Sastry are with the Department of Electrical Engineering and Computer Sciences, University of California, Berkeley, CA 94720-1774, U.S.A. Email: \{simic, zhangjun, sastry\}@eecs.berkeley.edu
}

tions of a hybrid automaton depend continuously on the initial state. Even though the class of hybrid systems that possess this property is known to be restricted [8], we feel that these conditions can be of general interest. For one thing, models that are sensitive to the choice of initial conditions are more difficult to simulate, or analyse numerically [9]. Furthermore, in this paper we show how, using continuity properties, one can derive an extension of LaSalle's principle for studying the stability of invariant sets to hybrid automata. This allows us to incorporate earlier results in this direction [10] in a more structured and general framework.

The paper is organised in five sections. The definitions of hybrid automata and executions are given in Section 2. In Section 3, continuity of executions with respect to initial conditions is defined and a class of systems that possessed this property is characterised. In Section 4, LaSalle's invariance principle is extended to hybrid automata. A summary and a discussion of ongoing work are given in Section 5. To avoid interrupting the flow of the paper the more straight-forward proofs have been omitted. The more important facts are proved in the appendix.

\section{Definitions and Notation}

Before we state the results of this paper, we recall some of the definitions and notation of [4]. For a finite collection $V$ of variables, let $\mathbf{V}$ denote the set of valuations (possible value assignments) of these variables. We use a lower case letter to denote both a variable and its valuation; the interpretation should be clear from the context. We refer to variables whose set of valuations is finite or countable as discrete, and to variables whose set of valuations is a subset of a Euclidean space as continuous. For a set of continuous variables $X$ with $\mathbf{X}=\mathbb{R}^{n}$ for some $n \geq 0$, we assume that $\mathbf{X}$ is given the Euclidean metric topology, and use $\|\cdot\|$ to denote the Euclidean norm. For a set of discrete variables $Q$, we assume that $\mathbf{Q}$ is given the discrete topology (every subset is an open set), generated by the metric $d_{D}\left(q, q^{\prime}\right)=0$ if $q=q^{\prime}$ and $d_{D}\left(q, q^{\prime}\right)=1$ if $q \neq q^{\prime}$. We denote the valuations of the union $Q \cup X$ by $\mathbf{Q} \times \mathbf{X}$, with the product topology generated by the metric

$$
d\left((q, x),\left(q^{\prime}, x^{\prime}\right)\right)=d_{D}\left(q, q^{\prime}\right)+\left\|x-x^{\prime}\right\| .
$$

We assume that a subset $U$ of a topological space is given the induced subset topology, and we use $\bar{U}$ to 

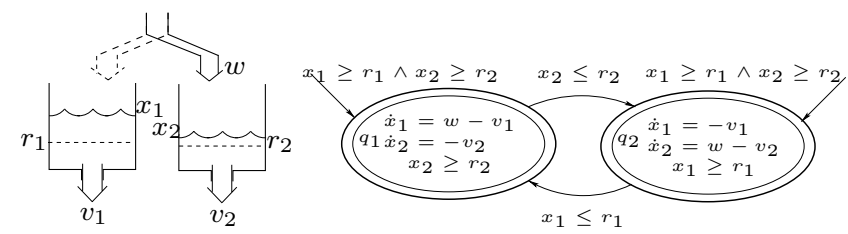

Figure 1: Water tank system and the corresponding hybrid automaton.

denote its closure, $U^{o}$ its interior, $\partial U=\bar{U} \backslash U^{o}$ its boundary, $U^{c}$ its complement, $|U|$ its cardinality, and $P(U)$ its power set (i.e., the set of all subsets of $U$ ). In logic formulas, we use $\wedge$ to denote "and".

A hybrid automaton is a dynamical system that describes the evolution in time of the valuations of a set of discrete and continuous variables.

Definition 2.1 $A$ hybrid automaton $H$ is a collection $H=(Q, X, f$, Init, $D, E, G, R)$, where

- $Q$ is a finite set of discrete variables;

- $X$ is a finite set of continuous variables;

- $f: \mathbf{Q} \times \mathbf{X} \rightarrow T \mathbf{X}$ is a vector field;

- Init $\subset \mathbf{Q} \times \mathbf{X}$ is a set of initial states;

- $D: \mathbf{Q} \rightarrow P(\mathbf{X})$ is a domain;

- $E \subset \mathbf{Q} \times \mathbf{Q}$ is a set of edges;

- $G: E \rightarrow P(\mathbf{X})$ is a guard condition;

- $R: E \times \mathbf{X} \rightarrow P(\mathbf{X})$ is a reset map.

We refer to $(q, x) \in \mathbf{Q} \times \mathbf{X}$ as the state of $H$. Because we are more interested in the discrete-continuous interplay than in the purely discrete or purely continuous dynamics, we impose the following standing assumption.

Assumption 2.1 The number of discrete states is finite $(|\mathbf{Q}|<\infty)$, and $\mathbf{X}=\mathbb{R}^{n}$, for some $n \geq 0$. For all $q \in \mathbf{Q}$, the vector field $f(q, \cdot)$ is globally Lipschitz continuous in its second argument. Moreover, for all $e \in E, G(e) \neq \emptyset$, and for all $x \in G(e), R(e, x) \neq \emptyset$.

It can be shown that the last part of the assumption can in fact be imposed without loss of generality [4].

It is sometimes convenient to visualise hybrid automata as directed graphs $(\mathbf{Q}, E)$ with vertices $\mathbf{Q}$ and edges $E$. The graphical notation is illustrated in the following example.

Example Consider the two-tank system of Alur and Henzinger [11] (Figure 1). For $i \in\{1,2\}$, let $x_{i}$ denote the volume of water in Tank $i$ and $v_{i}>0$ denote the constant flow of water out of Tank $i$. Let $w$ denote the constant flow of water into the system, dedicated exclusively to either Tank 1 or Tank 2 at each time instant.
The objective is to keep the water volumes above $r_{1}$ and $r_{2}$, respectively, assuming that the water volumes are above $r_{1}$ and $r_{2}$ initially. This is to be achieved by a controller that switches the inflow to Tank 1 whenever $x_{1} \leq r_{1}$ and to Tank 2 whenever $x_{2} \leq r_{2}$. The hybrid automaton describing this system is

- $Q=\{q\}$ with $\mathbf{Q}=\left\{q_{1}, q_{2}\right\}$

- $X=\left\{x_{1}, x_{2}\right\}$ with $\mathbf{X}=\mathbb{R}^{2}$;

- $f\left(q_{1}, x\right)=\left(w-v_{1},-v_{2}\right)$, $f\left(q_{2}, x\right)=\left(-v_{1}, w-v_{2}\right)$

- Init $=\mathbf{Q} \times\left\{x \in \mathbb{R}^{2}: x_{1} \geq r_{1} \wedge x_{2} \geq r_{2}\right\}$;

- $D\left(q_{1}\right)=\left\{x \in \mathbb{R}^{2}: x_{2} \geq r_{2}\right\}$ $D\left(q_{2}\right)=\left\{x \in \mathbb{R}^{2}: x_{1} \geq r_{1}\right\}$

- $E=\left\{\left(q_{1}, q_{2}\right),\left(q_{2}, q_{1}\right)\right\}$

- $G\left(q_{1}, q_{2}\right)=\left\{x \in \mathbb{R}^{2}: x_{2} \leq r_{2}\right\}$, $G\left(q_{2}, q_{1}\right)=\left\{x \in \mathbb{R}^{2}: x_{1} \leq r_{1}\right\}$

- $R\left(q_{1}, q_{2}, x\right)=R\left(q_{2}, q_{1}, x\right)=\{x\}$.

A hybrid time trajectory defines the time horizon of an execution of a hybrid automaton.

Definition 2.2 $A$ hybrid time trajectory is a finite or infinite sequence of intervals $\tau=\left\{I_{i}\right\}_{i=0}^{N}$, such that

- $I_{i}=\left[\tau_{i}, \tau_{i}^{\prime}\right]$ for all $i<N$;

- if $N<\infty$ then either $I_{N}=\left[\tau_{N}, \tau_{N}^{\prime}\right]$ or $I_{N}=$ $\left[\tau_{N}, \tau_{N}^{\prime}\right) ;$ and

- $\tau_{i} \leq \tau_{i}^{\prime}=\tau_{i+1}$ for all $i$.

Note that the right endpoint of one interval coincides with the left endpoint of the following interval. The interpretation is that these are the times at which discrete transitions take place. Note also that $\tau_{i}=\tau_{i}^{\prime}$ is allowed, therefore multiple discrete transitions may take place at the same time. Since all hybrid automata discussed here are time invariant we assume that $\tau_{0}=0$ without loss of generality.

Hybrid time trajectories can extend to infinity if $\tau$ is an infinite sequence or if it is a finite sequence ending with an interval of the form $\left[\tau_{N}, \infty\right)$. Each hybrid time trajectory $\tau$ is linearly ordered by the relation $\prec$, defined by $t_{1} \prec t_{2}$ for $t_{1} \in\left[\tau_{i}, \tau_{i}^{\prime}\right]$ and $t_{2} \in\left[\tau_{j}, \tau_{j}^{\prime}\right]$ if $t_{1}<t_{2}$ or $i<j$. We say that $\tau=\left\{I_{i}\right\}_{i=0}^{N}$ is a prefix of $\tau^{\prime}=\left\{J_{i}\right\}_{i=0}^{M}$ and write $\tau \leq \tau^{\prime}$ if either they are identical, or $\tau$ is finite, $N \leq M, I_{i}=J_{i}$ for all $i=0, \ldots$, $N-1$, and $I_{N} \subset J_{N}$. The prefix relation is a partial order on the set of all hybrid time trajectories.

For a hybrid time trajectory $\tau=\left\{I_{i}\right\}_{i=0}^{N}$, let $\langle\tau\rangle$ denote the set $\{0,1, \ldots, N\}$ if $N$ is finite and $\{0,1, \ldots\}$ if $N=\infty$. We use $q$ and $x$ to denote, respectively, the evolution of the discrete and continuous state over $\tau$. $q$ is a map from $\langle\tau\rangle$ to $\mathbf{Q}$ and $x=\left\{x^{i}: i \in\langle\tau\rangle\right\}$ is a collection of differentiable maps. An execution is now defined as a triple $\chi=(\tau, q, x)$ in the following way. 

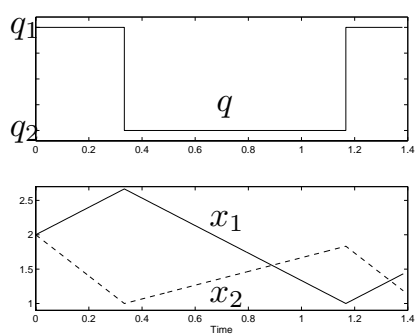

Figure 2: Example of an execution of the water tank hybrid automaton.

Definition 2.3 An execution of a hybrid automaton $H$ is a collection $\chi=(\tau, q, x)$, where $\tau$ is a hybrid time trajectory, $q:\langle\tau\rangle \rightarrow \mathbf{Q}$, and $x=\left\{x^{i}: i \in\langle\tau\rangle\right\}$ is a collection of differentiable maps $x^{i}: I_{i} \rightarrow \mathbf{X}$, with

- $\left(q(0), x^{0}(0)\right) \in$ Init;

- for all $t \in\left[\tau_{i}, \tau_{i}^{\prime}\right), \dot{x}^{i}(t)=f\left(q(i), x^{i}(t)\right)$ and $x^{i}(t) \in D(q(i)) ;$ and

- for all $i \in\langle\tau\rangle \backslash\{N\}, e=(q(i), q(i+1)) \in E$, $x^{i}\left(\tau_{i}^{\prime}\right) \in G(e)$, and $x^{i+1}\left(\tau_{i+1}\right) \in R\left(e, x^{i}\left(\tau_{i}^{\prime}\right)\right)$.

An example of an execution of the water tank automaton is shown in Figure 2.

We say that a hybrid automaton $H$ accepts an execution $\chi$ if $\chi$ fulfils the conditions of Definition 2.3. For an execution $\chi=(\tau, q, x)$, we use $\left(q_{0}, x_{0}\right)=\left(q\left(\tau_{0}\right), x^{0}\left(\tau_{0}\right)\right)$ to denote the initial state. The execution time $\mathcal{T}(\chi)$ is defined as

$$
\mathcal{T}(\chi)=\sum_{i=0}^{N}\left(\tau_{i}^{\prime}-\tau_{i}\right)=\lim _{i \rightarrow N} \tau_{i}^{\prime}-\tau_{0} .
$$

We say that an execution, $\chi=(\tau, q, x)$, of $H$ is a prefix of another execution, $\hat{\chi}=(\hat{\tau}, \hat{q}, \hat{x})$, of $H$ (write $\chi \leq \hat{\chi})$, if $\tau \leq \hat{\tau}$ and for all $i \in\langle\tau\rangle$ and all $t \in I_{i},\left(q(i), x^{i}(t)\right)=$ $\left(\hat{q}(i), \hat{x}^{i}(t)\right)$. We say $\chi$ is a strict prefix of $\hat{\chi}$ (write $\chi<$ $\hat{\chi})$, if $\chi \leq \hat{\chi}$ and $\chi \neq \hat{\chi}$. An execution is called maximal if it is not a strict prefix of any other execution. An execution is called finite if $\tau$ is a finite sequence ending with a compact interval, it is called infinite if $\tau$ is either an infinite sequence, or if $\mathcal{T}(\chi)=\infty$, and it is called Zeno if it is infinite but $\mathcal{T}(\chi)<\infty$.

We use $\mathcal{E}_{H}\left(q_{0}, x_{0}\right)$ to denote the set of all executions of $H$ with initial condition $\left(q_{0}, x_{0}\right) \in \operatorname{Init}, \mathcal{E}_{H}^{M}\left(q_{0}, x_{0}\right)$ to denote the set of all maximal executions, $\mathcal{E}_{H}^{*}\left(q_{0}, x_{0}\right)$ to denote the set of all finite executions, and $\mathcal{E}_{H}^{\infty}\left(q_{0}, x_{0}\right)$ to denote the set of all infinite executions. We use $\mathcal{E}_{H}$ to denote the union of $\mathcal{E}_{H}\left(q_{0}, x_{0}\right)$ over all $\left(q_{0}, x_{0}\right) \in$ Init.

Definition 2.4 A hybrid automaton $H$ is called nonblocking if $\mathcal{E}_{H}^{\infty}\left(q_{0}, x_{0}\right)$ is non-empty for all $\left(q_{0}, x_{0}\right) \in$ Init. It is called deterministic if $\mathcal{E}_{H}^{M}\left(q_{0}, x_{0}\right)$ contains at most one element for all $\left(q_{0}, x_{0}\right) \in$ Init.
Conditions for determining whether general hybrid automata are deterministic and/or non-blocking are given in [4]. Algorithmic conditions for special classes of hybrid automata can be found in $[2,12]$ (for complementarity systems) and $[13,14]$ (for piecewise linear systems).

The continuity conditions developed in the next sections involve the set of states reachable by a hybrid automaton and the set of states from which continuous evolution is impossible. The set of states reachable by $H$, Reach $_{H}$, is given by

$$
\begin{aligned}
\operatorname{Reach}_{H}=\left\{(\hat{q}, \hat{x}) \in \mathbf{Q} \times \mathbf{X}: \exists \chi=(\tau, q, x) \in \mathcal{E}_{H}^{*},\right. \\
\\
\left.\left(q(N), x^{N}\left(\tau_{N}^{\prime}\right)\right)=(\hat{q}, \hat{x})\right\} .
\end{aligned}
$$

Clearly, Init $\subset$ Reach $_{H}$, since we may choose $N=0$ and $\tau_{0}^{\prime}=\tau_{0}$.

The set of states from which continuous evolution is impossible is given by

$$
\begin{aligned}
\mathrm{Out}_{H}= & \{(q, x) \in \mathbf{Q} \times \mathbf{X}: \forall \epsilon>0, \exists t \in[0, \epsilon), \\
& \psi(t, q, x) \notin D(q)\} .
\end{aligned}
$$

Note that $\{q\} \times D(q)^{c} \subset \mathrm{Out}_{H}$. Moreover, if $D(q)$ is an open set, then $\left\{x \in \mathbf{X}:(q, x) \in \operatorname{Out}_{H}\right\}=D(q)^{c}$. However, if $D(q)$ is closed for some $q \in \mathbf{Q}$, then Out $_{H}$ may also contain parts of the boundary of $D(q)$. For certain classes of hybrid automata the computation of Out $_{H}$ and Reach $_{H}$ is straightforward [4]. Reachability computations are, however, difficult in general.

Notice that Definition 2.3 does not require the state to remain in the domain. The state may start outside the domain, if for some $(q, x) \in \mathrm{I}$ it,$x \notin D(q)$. Moreover, if a domain $D(q)$ is open, the state can reach a point in $\overline{D(q)} \backslash D(q)$ by flowing along $f(q, \cdot)$. Finally, the state can take a discrete transition from some $(q, x)$ with $x \in$ $D(q)$ to some $\left(q^{\prime}, x^{\prime}\right)$ with $\left(q, q^{\prime}\right) \in E, x \in G\left(q, q^{\prime}\right)$, and $x^{\prime} \in R\left(\left(q, q^{\prime}\right), x\right) \cap D^{c}\left(q^{\prime}\right)$. To prevent this situation we impose an additional standing assumption. Let

$$
\operatorname{Dom}_{H}=\bigcup_{q \in \mathbf{Q}}\{q\} \times D(q) \subset \mathbf{Q} \times \mathbf{X} .
$$

We call an automaton $H$ domain preserving if $\operatorname{Reach}_{H} \subset \operatorname{Dom}_{H}$.

Assumption 2.2 $\operatorname{Dom}_{H}$ is closed. The hybrid automata considered are domain preserving, with $\mathrm{I} n i t=$ $\operatorname{Dom}_{H}$.

The assumption that $H$ is domain preserving is the most important part of Assumption 2.2. Even though it may seem restrictive, it often turns out to be implicit in models of physical systems, where the domains are typically used to encode physical constraints that all executions of the system must satisfy. The assumption 
that $\operatorname{Dom}_{H}$ is closed can be relaxed for much of the subsequent discussion, by changing the definitions to include the closures of appropriate sets. The assumption that $\mathrm{I}$ it $=\operatorname{Dom}_{H}$ is only made for convenience, to avoid having to argue whether certain states in the domain are reachable. Subsequent proofs are still valid if the conditions of the theorems hold for all states in Reach $_{H}$ (as opposed to all states in $\operatorname{Dom}_{H}$ ). Notice that, under Assumption 2.2, $\operatorname{Reach}_{H}=\operatorname{Dom}_{H}$.

Determining whether a hybrid automaton satisfies Assumption 2.2 is usually straightforward. Using an induction argument on the length of the executions one can show the following.

Lemma 2.1 Consider a hybrid automaton $H$ such that the set $\operatorname{Dom}_{H}$ is closed. $H$ is domain preserving if Init $\subset \operatorname{Dom}_{H}$ and $R\left(\left(q, q^{\prime}\right), x\right) \subset D\left(q^{\prime}\right)$ for all $q \in \mathbf{Q}$, all $\left(q, q^{\prime}\right) \in E$ and all $x \in D(q) \cap G\left(q, q^{\prime}\right)$.

Using the conditions of Lemma 2.1, one can show that the water tank system is domain preserving.

\section{Continuity with Initial Conditions}

In general, the behaviour of hybrid automata may change dramatically even for small changes in initial conditions. This fact is unavoidable, if one wants to allow hybrid automata that are powerful enough to model realistic systems. However, discontinuous dependence on initial conditions may cause problems, both theoretical and practical, when one tries to simulate hybrid automata [8, 9]. Motivated by this observation, a number of authors have investigated continuity with respect to initial conditions. In [8] it was shown that a reasonably large class of hybrid automata is continuous for almost all initial conditions. In [15] a Skorohod topology was proposed as a framework for formulating continuity properties. Even though this topology (initially developed for the space of piecewise continuous functions) is natural for studying continuity in hybrid systems, the definition and properties of the Skorohod metric make it difficult to work with in practice. Here we give the following alternative (and we feel easier to work with) definition of continuity.

Definition 3.1 A hybrid automaton $H$ is called continuous if for all finite executions $\chi=(\tau, q, x) \in$ $\mathcal{E}_{H}^{*}\left(q_{0}, x_{0}\right)$ with $\tau=\left\{I_{i}\right\}_{i=0}^{N}$ and all $\epsilon>0$, there exists $\delta>0$ such that all maximal executions in $\mathcal{E}_{H}^{M}\left(\tilde{q}_{0}, \tilde{x}_{0}\right)$ with $d\left(\left(\tilde{q}_{0}, \tilde{x}_{0}\right),\left(q_{0}, x_{0}\right)\right)<\delta$ have a finite prefix $\tilde{\chi}=$ $(\tilde{\tau}, \tilde{q}, \tilde{x}) \in \mathcal{E}_{H}^{*}\left(\tilde{q}_{0}, \tilde{x}_{0}\right)$ with $\tilde{\tau}=\left\{\tilde{I}_{i}\right\}_{i=0}^{N}$ that satisfies

$$
\begin{aligned}
& \text { 1. }|\mathcal{T}(\tilde{\chi})-\mathcal{T}(\chi)|<\epsilon \text {; and } \\
& \text { 2. } d\left(\left(\tilde{q}(N), \tilde{x}^{N}\left(\tilde{\tau}_{N}^{\prime}\right)\right),\left(q(N), x^{N}\left(\tau_{N}^{\prime}\right)\right)\right)<\epsilon .
\end{aligned}
$$

Roughly speaking, $H$ is continuous if two executions starting close to one another remain close to one another. Notice that if $H$ is continuous, one can choose $\delta$ such that finite executions starting within $\delta$ of one another go through the same sequence of discrete transitions.

The following theorem provides conditions under which a hybrid automaton is guaranteed to be continuous.

Theorem 3.1 A hybrid automaton $H$ is continuous if

1. $H$ is deterministic;

2. for all $e=\left(q, q^{\prime}\right) \in E, G(e) \cap D(q)$ is an open subset of $\partial D(q)$;

3. for all $e \in E, R(e, \cdot)$ is a continuous function;

4. there exists a function $\sigma: \mathbf{Q} \times \mathbf{X} \rightarrow \mathbb{R}$, differentiable in its second argument, such that for all $q \in \mathbf{Q}, D(q)=\{x \in \mathbf{X} \mid \sigma(q, x) \geq 0\}$;

5. for all $(q, x)$ with $\sigma(q, x)=0, L_{f} \sigma(q, x) \neq 0$.

Roughly speaking, conditions 4 and 5 are used to show that if from some initial state we can flow to a state from which a discrete transition is possible, then from all neighbouring states we can do the same. This observation is summarised in the following lemma.

Lemma 3.1 Consider a hybrid automaton, $H$, satisfying conditions 4 and 5 of Theorem 3.1. Let $\chi=$ $(\tau, q, x) \in \mathcal{E}_{H}\left(q_{0}, x_{0}\right)$ be a finite execution of $H$ defined over an interval $\tau=\left[0, \tau_{0}^{\prime}\right]$ with $\tau_{0}^{\prime}>0$ and $x^{0}\left(\tau_{0}^{\prime}\right) \in$ $\partial D\left(q_{0}\right)$. Then there exists a neighbourhood $W \subset D\left(q_{0}\right)$ of $x_{0}$ and a differentiable function $T: W \rightarrow \mathbb{R}^{+}$, such that for all $y \in W$,

1. $\psi\left(T(y), q_{0}, y\right) \in \partial D\left(q_{0}\right)$;

2. $\psi\left(t, q_{0}, y\right) \in D\left(q_{0}\right)^{o}$ for all $t \in(0, T(y))$; and

3. $\Psi: W \rightarrow \partial D\left(q_{0}\right)$, defined by $\Psi(y)=$ $\psi\left(T(y), q_{0}, y\right)$, is continuous.

To complete the proof of Theorem 3.1, conditions 1, 2 and 3 are used to piece together the intervals of continuous evolution. The details are given in the appendix.

It is easy to check that the water tank automaton satisfies the conditions of Theorem 3.1, and therefore is continuous.

\section{Stability of Invariant Sets}

We first recall some standard concepts from dynamical system theory, and discuss how they extend to hybrid automata.

Definition 4.1 $A$ set $M \subset$ Reach $_{H}$ is called invariant if for all $\left(q_{0}, x_{0}\right) \in M$, and all $(\tau, q, x) \in$ $\mathcal{E}_{H}\left(q_{0}, x_{0}\right),\left(q(i), x^{i}(t)\right) \in M$ for all $i \in\langle\tau\rangle$ and $t \in I_{i}$. 
Clearly, the class of invariant sets is closed under arbitrary unions and intersections.

The asymptotic behaviour of an infinite execution is captured by its $\omega$-limit set.

Definition 4.2A point $(\hat{q}, \hat{x}) \in \mathbf{Q} \times \mathbf{X}$ is an $\omega$-limit point of an infinite execution $\chi=(\tau, q, x) \in \mathcal{E}_{H}^{\infty}$, if there exists a sequence $\left\{\theta_{n}\right\}_{n=0}^{\infty}$ with $\theta_{n} \in I_{i_{n}}$ and $i_{n} \in\langle\tau\rangle$ such that as $n \rightarrow \infty, \theta_{n} \rightarrow \mathcal{T}(\chi)$ and $\left(q\left(i_{n}\right), x^{i_{n}}\left(\theta_{n}\right)\right) \rightarrow(\hat{q}, \hat{x})$. The $\omega$-limit set, $S_{\chi} \subset$ $\mathbf{Q} \times \mathbf{X}$, of $\chi \in \mathcal{E}_{H}^{\infty}$ is the set of all $\omega$-limit points of $\chi$.

Notice that under Assumption 2.2, $\omega$-limit points are reachable. The following proposition establishes some basic properties of $\omega$-limit sets for deterministic, continuous hybrid automata.

Lemma 4.1 Let $H$ be a deterministic, continuous hybrid automaton. Consider an infinite execution $\chi=$ $(\tau, q, x)$ and assume that there exists $C>0$ such that for all $i \in\langle\tau\rangle$ and all $t \in\left[\tau_{i}, \tau_{i}^{\prime}\right],\left\|x^{i}(t)\right\|<C$. Then the $\omega$-limit set $S_{\chi}$ of $\chi$ is a nonempty, compact, invariant set. Furthermore, for all $\epsilon>0$ there exists $K \in\langle\tau\rangle$ such that $d\left(\left(q(t), x^{n}(t)\right), S_{\chi}\right)<\epsilon$ for all $n>K$ and $t \in I_{n}$.

The proof is an extension of the corresponding proofs for continuous dynamical systems (see for example [16]). The details are rather tedious and are omitted.

LaSalle's invariance principle for continuous dynamical systems [17] provides conditions for an invariant set to be attracting. The following statement extends the result to continuous hybrid automata.

Theorem 4.1 Consider a non-blocking, deterministic and continuous hybrid automaton $H$. Let $\Omega \subset$ Reach $_{H}$ be a compact invariant set and define $\Omega_{1}=\Omega \cap \mathrm{Out}_{H}^{c}$ and $\Omega_{2}=\Omega \cap$ Out $_{H}$. Assume there exists a continuous function $V: \Omega \rightarrow \mathbb{R}$, such that

1. for all $(q, x) \in \Omega_{1}, V$ is continuously differentiable with respect to $x$ and $L_{f} V(q, x) \leq 0$,

2. for all $(q, x) \in \Omega_{2}, e=\left(q, q^{\prime}\right) \in E$, $V\left(q^{\prime}, R(e, x)\right) \leq V(q, x)$.

Define $S_{1}=\left\{(q, x) \in \Omega_{1}: L_{f} V(q, x)=0\right\}$ and $S_{2}=\left\{(q, x) \in \Omega_{2}: \forall e=\left(q, q^{\prime}\right) \in E, V\left(q^{\prime}, R(e, x)\right)=\right.$ $V(q, x)\}$. Let $M$ be the largest invariant subset of $S_{1} \cup S_{2}$. Then, for all $\left(q_{0}, x_{0}\right) \in \Omega$ the execution $\chi=(\tau, q, x) \in \mathcal{E}_{H}^{\infty}\left(q_{0}, x_{0}\right)$ approaches $M$ as $t \rightarrow \mathcal{T}(\chi)$.

"Approaches" should be interpreted as $\lim _{t \rightarrow \mathcal{T}(\chi)} d\left(\left(q(t), x^{i}(t)\right), M\right)=0$. Note that since the class of invariant sets is closed under arbitrary unions, $M$, the unique largest invariant set contained in $S_{1} \cup S_{2}$, exists.

We demonstrate the use of this extension of LaSalle's invariance principle on the water tank system.

Example Consider the water tank hybrid automaton and assume that $r_{1}=r_{2}=0$, and $\max \left(v_{1}, v_{2}\right)<w<$ $v_{1}+v_{2}$. It is easy to show that this is a non-blocking, deterministic, continuous, Zeno hybrid automaton [5]. Consider the set

$$
\begin{aligned}
& \Omega=\left\{q_{1}, q_{2}\right\} \times\left\{x \in \mathbb{R}^{2}: x_{1} \geq 0 \wedge x_{2} \geq 0 \wedge\right. \\
& \left.\quad \max \left\{\left(w-v_{2}\right) x_{1}+v_{1} x_{2}, v_{2} x_{1}+\left(w-v_{1}\right) x_{2}\right\} \leq K\right\}
\end{aligned}
$$

for an arbitrary $K>0$. Clearly $\Omega$ is compact. One can also show that $\Omega$ is invariant, by induction on the length of the system executions. A straight-forward computation reveals that

$$
\begin{aligned}
\Omega_{2}= & \left\{q_{1}\right\} \times\left\{x \in \mathbb{R}^{2}:\right. \\
& \left.x_{1} \in\left[0, \min \left\{K /\left(w-v_{2}\right), K / v_{2}\right\}\right] \wedge x_{2}=0\right\} \cup \\
& \left\{q_{2}\right\} \times\left\{x \in \mathbb{R}^{2}:\right. \\
& \left.x_{1}=0 \wedge x_{2} \in\left[0, \min \left\{K /\left(w-v_{1}\right), K / v_{1}\right\}\right]\right\} .
\end{aligned}
$$

By definition, $\Omega_{1}=\Omega \backslash \Omega_{2}$.

Let $V(q, x)=x_{1}+x_{2}$. Then for all $(q, x) \in \Omega_{1}$, $L_{f} V(q, x)=w-\left(v_{1}+v_{2}\right)<0$. Therefore, condition 1 of Theorem 4.1 is satisfied and $S_{1}=\emptyset$. Moreover, since $R$ is the identity, $V\left(q^{\prime}, R\left(\left(q, q^{\prime}\right), x\right)=V(q, x)\right.$ whenever a transition is possible. Therefore, condition 2 of Theorem 4.1 is satisfied and $S_{2}=\Omega_{2}$. Notice that the set $\left\{q_{1}, q_{2}\right\} \times\{(0,0)\}$ is invariant. Moreover, this is the only (hence maximal) invariant subset of $S_{2}$, since if either $x_{1}>0$ or $x_{2}>0$, after the discrete transition continuous evolution would be initiated, taking the state out of $\Omega_{2}$. Therefore, from Theorem 4.1, for all $\left(q_{0}, x_{0}\right) \in \Omega$ the execution $\chi=(\tau, q, x) \in \mathcal{E}_{H}^{\infty}\left(q_{0}, x_{0}\right)$ converges to $\left\{q_{1}, q_{2}\right\} \times\{(0,0)\}$ as $t \rightarrow \mathcal{T}(\chi)$.

Notice that since $K$ can be taken arbitrarily large in the example, the set $\left\{q_{1}, q_{2}\right\} \times\{(0,0)\}$ is, in a sense, globally attracting. The conclusion of the example could also have been derived using the properties of Zeno executions established in [18]. The advantage of using LaSalle's principle is that it does not require one to integrate the differential equations and argue about their solutions, which is needed, for example, to establish that the system is Zeno.

\section{Conclusions}

An definition of continuous dependence of the executions of a hybrid automaton with respect to initial conditions was given. Conditions were derived to establish 
a class of hybrid automata that possess this continuity property. For this class of automata, an extension of LaSalle's principle for studying the stability of invariant sets was derived.

We view the results in this paper as one more step towards the analysis of hybrid systems from a dynamical systems perspective. We are currently pursuing this line of work further by research into methods for dealing with systems with inputs. This will allow one to model progressively more complicated systems through the composition (appropriately defined) of simpler components.

\section{References}

[1] A. S. Morse, "Control using logic-based switching", in Trends in Control. A European Perspective, A. Isidori, Ed., pp. 69-113. Springer-Verlag, 1995.

[2] A. J. van der Schaft and J. M. Schumacher, "Complementarity modeling of hybrid systems", IEEE Transactions on Automatic Control, vol. 43, no. 4, pp. 483-490, April 1998.

[3] M. Johansson, Piecewise linear control systems, PhD thesis, Department of Automatic Control, Lund Institute of Technology, Sweden, March 1999.

[4] J. Lygeros, K. H. Johansson, S. Sastry, and M. Egerstedt, "On the existence of executions of hybrid automata", in IEEE Conference on Decision and Control, Phoenix, AZ, 1999, pp. 2249-2254.

[5] K. H. Johansson, M. Egerstedt, J. Lygeros, and S. Sastry, "On the regularization of Zeno hybrid automata", System \& Control Letters, vol. 38, pp. 141150, 1999.

[6] M. Egerstedt, K. H. Johansson, J. Lygeros, and S. Sastry, "On the existence of executions of hybrid automata", in IEEE Conference on Decision and Control, Phoenix, Arizona, U.S.A., December 7-10 1999, pp. 2249-2254.

[7] S. Simić, K. H. Johansson, S. Sastry, and J. Lygeros, "Towards a geometric theory of hybrid systems", in Hybrid Systems: Computation and Control, B. Krogh and N. Lynch, Eds., vol. 1790 of Lecture Notes in Computer Science, pp. 421-436. SpringerVerlag, 2000.

[8] L. Tavernini, "Differential automata and their discrete simulators", Nonlinear Analysis, Theory, Methods \& Applications, vol. 11, no. 6, pp. 665-683, 1987.

[9] K. H. Johansson, J. Lygeros, S. Sastry, and M. Egerstedt, "Simulation of Zeno hybrid automata", in Proc. 38th IEEE Conference on Decision and Control, Phoenix, AZ, 1999, pp. 3538-3543.

[10] J. Zhang, K. H. Johansson, J. Lygeros, and S. Sastry, "Dynamical systems revisited: Hybrid sys- tems with Zeno executions", in Hybrid Systems: Computation and Control, B. Krogh and N. Lynch, Eds., vol. 1790 of Lecture Notes in Computer Science, pp. 451-464. Springer-Verlag, 2000.

[11] R. Alur and T. A. Henzinger, "Modularity for timed and hybrid systems", in CONCUR 97: Concurrency Theory, Lecture Notes in Computer Science 1243, pp. 74-88. Springer-Verlag, 1997.

[12] M. Heemels, Linear Complementarity Systems, PhD thesis, University of Einhoven, 1999.

[13] J. Imura and A. J. van der Schaft, "Characterization of well-posedness of piecewise linear systems", IEEE Transactions on Automatic Control, vol. 45, no. 9, pp. 1600-1619, September 2000.

[14] M. Lemmon, "On the existence of solutions to controlled hybrid automata", in Hybrid Systems: Computation and Control, B. Krogh and N. Lynch, Eds., vol. 1790 of Lecture Notes in Computer Science, pp. 229-242. Springer-Verlag, 2000.

[15] M. Broucke, "Regularity of solutions and homotopy equivalence for hybrid systems", in IEEE Conference on Decision and Control, Tampa, FL, 1998.

[16] S. Sastry, Nonlinear Systems: Analysis, Stability, and Control, Springer-Verlag, NY, 1999.

[17] J. P. LaSalle, "Stability theory for ordinary differential equations", J. Diff. Eq., vol. 4, pp. 57-65, 1968.

[18] J. Zhang, K. H. Johansson, J. Lygeros, and S. Sastry, "Zeno hybrid systems", International Journal of Nonlinear and Robust Control, 2000, Accepted for publication.

[19] F.H. Clarke, Yu.S. Ledyaev, R.J. Stern, and P.R. Wolenski, Nonsmooth analysis and control theory, Springer Verlag, New York, 1998.

Proof of Lemma 3.1: Since $\tau_{0}^{\prime}>0$, the state $\left(q_{0}, x^{0}\left(\tau_{0}^{\prime}\right)\right)$ is reached from $\left(q_{0}, x_{0}\right)$ along continuous evolution. We drop the superscript on $x$ to simplify the notation.

To show 1, notice that, by the definition of an execution, $x(t) \in D\left(q_{0}\right)$ for all $t \in\left[\tau_{i}, \tau_{i}^{\prime}\right)$. Since $x\left(\tau_{0}^{\prime}\right) \in \partial D\left(q_{0}\right), \sigma\left(q_{0}, x\left(\tau_{0}^{\prime}\right)\right)=0$. The composed function $\sigma\left(q_{0}, \psi\left(\cdot, q_{0}, \cdot\right)\right)$ is differentiable in its first argument $(t)$ in a neighbourhood of $\left(\tau_{0}^{\prime}, x_{0}\right)$ in $\mathbb{R}^{+} \times \mathbb{R}^{n}$. This is because by assumption $\sigma\left(q_{0}, \cdot\right)$ is differentiable and the flow $\psi\left(\cdot, q_{0}, \cdot\right)$ is differentiable in its first argument. Moreover, $\sigma\left(q_{0}, \psi\left(\cdot, q_{0}, \cdot\right)\right)$ is continuous in its second argument $(x)$ in a neighbourhood of $\left(\tau_{0}^{\prime}, x_{0}\right)$ in $\mathbb{R}^{+} \times \mathbb{R}^{n}$. This is because $\sigma\left(q_{0}, \cdot\right)$ is continuous and $\psi\left(\cdot, q_{0}, \cdot\right)$ is continuous in its second argument (by Assumption 2.1). Finally,

$$
\left.\frac{\partial}{\partial t} \sigma\left(q_{0}, \psi\left(t, q_{0}, x\right)\right)\right|_{(t, x)=\left(\tau_{0}^{\prime}, x_{0}\right)}=L_{f} \sigma\left(q_{0}, x\left(\tau_{0}^{\prime}\right)\right) \neq 0
$$


(by condition 5 of Theorem 3.1). By the Implicit Function Theorem (in particular, the non-smooth version found in [19], Theorem 3.3.6), there exists a neighbourhood $\Omega \subset \mathbb{R}^{+}$of $\tau_{0}^{\prime}$ and a neighbourhood $W \subset$ $\mathbb{R}^{n}$ of $x_{0}$, such that for each $y \in W$ the equation $\sigma\left(q_{0}, \psi\left(t, q_{0}, y\right)\right)=0$ has a unique solution $t \in \Omega$. Furthermore, this solution is given by $t=T(y)$, where $T$ is a continuous mapping from $W$ to $\Omega$ and $\psi\left(T(y), q_{0}, y\right) \in$ $\partial D\left(q_{0}\right)$.

To show 2, assume that for some $y \in W$ there exists $t \in(0, T(y))$ such that $\psi\left(t, q_{0}, y\right) \in \partial D(q(i))$. Then, $\sigma\left(q_{0}, \psi\left(t, q_{0}, y\right)\right)=0$, which contradicts the fact that $T(y)$ is the unique solution to the equation $\sigma\left(q_{0}, \psi\left(t, q_{0}, y\right)\right)=0$.

Finally, to show 3 , recall that, since $\psi\left(\cdot, q_{0}, \cdot\right)$ is continuous in both arguments, for all $\epsilon>0$ there exists $\delta_{1}>0$, such that for all $t$ with $\left\|t-T\left(x_{0}\right)\right\|<\delta_{1}$ and all $y \in W$ with $\left\|y-x_{0}\right\|<\delta_{1}$,

$$
\begin{gathered}
\left\|\psi\left(t, q_{0}, x_{0}\right)-\psi\left(T\left(x_{0}\right), q_{0}, x_{0}\right)\right\|<\epsilon \\
\left\|\psi\left(T(y), q_{0}, y\right)-\psi\left(T(y), q_{0}, x_{0}\right)\right\|<\epsilon .
\end{gathered}
$$

By the continuity of $T$ there exists some $\delta_{2}>0$ such that for all $y \in W$ with $\left\|y-x_{0}\right\|<\delta_{2}$, we have $\| T(y)-$ $T\left(x_{0}\right) \|<\delta_{1}$. By setting $\delta=\min \left(\delta_{1}, \delta_{2}\right)$, it follows that for all $y \in W$ with $\left\|y-x_{0}\right\|<\delta$,

$$
\begin{aligned}
\left\|\Psi(y)-\Psi\left(x_{0}\right)\right\|= & \left\|\psi\left(T(y), q_{0}, y\right)-\psi\left(T\left(x_{0}\right), q_{0}, x_{0}\right)\right\| \\
\leq & \left\|\psi\left(T(y), q_{0}, y\right)-\psi\left(T(y), q_{0}, x_{0}\right)\right\| \\
& +\left\|\psi\left(T(y), q_{0}, x_{0}\right)-\psi\left(T\left(x_{0}\right), q_{0}, x_{0}\right)\right\| \\
< & 2 \epsilon,
\end{aligned}
$$

which proves the continuity of $\Psi$.

The proof of Theorem 3.1 also makes use of the following fact.

Proof of Theorem 3.1: Consider a finite execution $\chi=(\tau, q, x) \in \mathcal{E}_{H}\left(q_{0}, x_{0}\right)$ with $\tau=\left\{I_{i}\right\}_{i=0}^{N}$ and an $\epsilon>0$. We construct a sequence of sets $\left\{W^{0}, V^{0}, \ldots, W^{N}, V^{N}\right\}$, where $W^{i} \subset D(q(i))$ is a neighbourhood of $x^{i}\left(\tau_{i}\right)$ and $V^{i} \subset D(q(i))$ is a neighbourhood of $x^{i}\left(\tau_{i}^{\prime}\right)$, such that the continuous evolution in $q(i)$ provides a continuous map from $W^{i}$ to $V^{i}$ and the reset $R\left(e_{i}, \cdot\right)$ provides continuous map from $V^{i}$ to $W^{i+1}$. The notation is illustrated in Figure 3 .

The construction is recursive, starting with $i=N$. Define $V^{N}=\left\{x \in D(q(N)):\left\|x-x^{N}\left(\tau_{N}^{\prime}\right)\right\|<\epsilon\right\}$. We distinguish the following three cases:

Case 1: $\tau_{N}^{\prime}>\tau_{N}$ and $x^{N}\left(\tau_{N}^{\prime}\right) \in \partial D(q(N)) . \quad$ By Lemma 3.1, there exists a neighbourhood, $W \subset D(q(N))$, of $x^{N}\left(\tau_{N}\right)$ and a differentiable function, $T: W \rightarrow \mathbb{R}^{+}$, such that for all $y \in W, \psi(T(y), q(N), y) \in \partial D(q(N))$ and

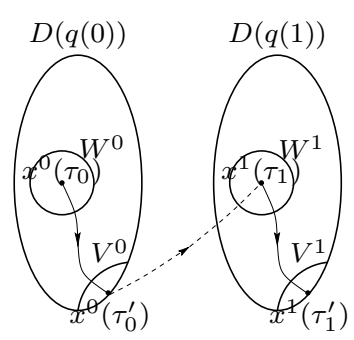

Figure 3: Illustration of the proof of Theorem 3.1 for $N=$ 1 and Case 1.

$\psi(t, q(N), y) \in D(q(N))^{o}$ for all $t \in(0, T(y))$. As in Lemma 3.1, define $\Psi^{N}: W \rightarrow \partial D(q(N))$ by $\Psi^{N}(y)=\psi(T(y), q(N), y)$. By the continuity of $\Psi^{N}$, there exists a neighbourhood, $W^{N} \subset W$, of $x^{N}\left(\tau_{N}\right)$ such that $\Psi^{N}\left(W^{N}\right) \subset V^{N}$. Furthermore, all executions $\tilde{\chi}$ with $\tilde{x}^{N}\left(\tilde{\tau}_{N}\right) \in W^{N}$ fulfil $\tilde{x}^{N}\left(\tilde{\tau}_{N}+T^{N}\left(\tilde{x}^{N}\left(\tilde{\tau}_{N}\right)\right)\right) \in V^{N}$.

Case 2: $\tau_{N}^{\prime}>\tau_{N}$ and $x^{N}\left(\tau_{N}^{\prime}\right) \in D(q(N))^{o}$. Define $T^{N}$ by $T^{N}(y) \equiv \tau_{N}^{\prime}-\tau_{N}$. Let $W \subset D(q(N))$ be a neighbourhood of $x^{N}\left(\tau_{N}\right)$ such that for all $y \in W$ and $t \in\left(0, \tau_{N}^{\prime}-\tau_{N}\right), \psi(t, q(N), y) \in D(q(N))^{o}$. Such a neighbourhood exists, because for all $t \in\left(0, \tau_{N}^{\prime}-\right.$ $\left.\tau_{N}\right), \quad \psi\left(t, q(N), x^{N}\left(\tau_{N}\right)\right) \in D(q(N))^{\circ}$ (cf., proof of Lemma 3.1). Define a function $\Psi^{N}: W \rightarrow D(q(N))$ by $\Psi^{N}(y)=\psi\left(T^{N}(y), q(N), y\right)$. By continuous dependence of the solutions of the differential equation with respect to initial conditions, there exists a neighbourhood $W^{N} \subset W$ of $x^{N}\left(\tau_{N}\right)$ such that both $\Psi^{N}\left(W^{N}\right) \subset$ $V^{N}$ and all executions with $\tilde{x}^{N}\left(\tilde{\tau}_{N}\right) \in W^{N}$ satisfy $\tilde{x}^{N}\left(\tilde{\tau}_{N}+T^{N}\left(\tilde{x}^{N}\left(\tilde{\tau}_{N}\right)\right)\right) \in V^{N}$.

Case 3: $\tau_{N}^{\prime}=\tau_{N}$. Define $T^{N}$ by $T^{N}(y) \equiv 0, W^{N}=$ $V^{N}$ and $\Psi^{N}$ the identity map. Clearly, $\Psi^{N}\left(W^{N}\right)=$ $V^{N}$.

Next let us define $V^{N-1}$. Let $e_{i}=(q(i), q(i+$ $1)$ ) and notice that $x^{N-1}\left(\tau_{N-1}^{\prime}\right) \in G\left(e_{N-1}\right)$. Since $R\left(e_{N-1}, \cdot\right)$ is continuous, there exists a neighbourhood $V \subset D(q(N-1))$ of $x^{N-1}\left(\tau_{N-1}^{\prime}\right)$ such that $R\left(e_{N-1}, V \cap G\left(e_{N-1}\right)\right) \subset W^{N}$. By condition 2 of the theorem, $G\left(e_{N-1}\right) \cap D(q(N-1))$ is an open subset of $\partial D(q(N-1))$, so there exists a neighbourhood $V^{N-1} \subset$ $V$ of $x^{N-1}\left(\tau_{N-1}^{\prime}\right)$ such that $V^{N-1} \cap \partial D(q(N-1)) \subset$ $G\left(e_{N-1}\right) \cap D(q(N-1))$. Since $H$ is deterministic, it follows that all executions with $\tilde{q}(N-1)=q(N-1)$ and $\tilde{x}^{N-1}\left(\tilde{\tau}_{N-1}^{\prime}\right) \in V^{N-1} \cap \partial D(q(N-1))$ satisfy $\tilde{x}^{N}\left(\tilde{\tau}_{N}\right) \in$ $W^{N}$.

Next, define $T^{N-1}$ and $\Psi^{N-1}$ using Lemma 3.1, as for Cases 1 and 3 above. There exists a neighbourhood $W^{N-1} \subset D(q(N-1))$ of $x^{N-1}\left(\tau_{N-1}\right)$ such that $\Psi^{N-1}\left(W^{N-1}\right) \subset V^{N-1} \cap \partial D(q(N-1))$. Moreover, all executions $\tilde{\chi}$ with $\tilde{x}^{N-1}\left(\tilde{\tau}_{N-1}\right) \in W^{N-1}$ satisfy $\tilde{x}^{N-1}\left(\tilde{\tau}_{N-1}^{\prime}\right) \in V^{N-1} \cap \partial D(q(N-1))$ and $\tilde{\tau}_{N-1}^{\prime}=$ $\tilde{\tau}_{N-1}+T^{N-1}\left(\tilde{x}^{N-1}\left(\tilde{\tau}_{N-1}\right)\right)$. If $\tau_{N-1}^{\prime}=\tau_{N-1}$, some 
executions close to $\chi$ may take an instantaneous transition from $q(N-1)$ to $q(N)\left(\tilde{\tau}_{N-1}^{\prime}=\tilde{\tau}_{N-1}\right)$ while others may have to flow for a while $\left(\tilde{\tau}_{N-1}^{\prime}>\tilde{\tau}_{N-1}\right)$ before they follow $\chi$ 's transition from $q(N-1)$ to $q(N)$. In the former case $T^{N-1}$ and $\Psi^{N-1}$ can be defined as in Case 3 above, while in the latter they can be defined as in Case 1.

By induction, we can construct a sequence of sets $\left\{W^{0}, V^{0}, \ldots, W^{N}, V^{N}\right\}$ and continuous functions $T^{i}$ : $W^{i} \rightarrow \mathbb{R}^{+}$and $\Psi^{i}: W^{i} \rightarrow V^{i}$ for $i=0, \ldots, N$. For $k=1, \ldots, N$, define the function $\Phi^{k}: W^{0} \rightarrow W^{k}$ recursively as

$$
\begin{aligned}
\Phi^{0}\left(\tilde{x}_{0}\right) & =\tilde{x}_{0} \\
\Phi^{k}\left(\tilde{x}_{0}\right) & =R\left(e_{k-1}, \Psi^{k-1}\left(\Phi^{k-1}\left(\tilde{x}_{0}\right)\right)\right)
\end{aligned}
$$

For $k=0, \ldots, N$, define the function $\gamma^{k}: W^{0} \rightarrow \mathbb{R}^{+}$as

$$
\gamma^{k}\left(\tilde{x}_{0}\right)=\sum_{\ell=0}^{k} T^{\ell}\left(\Phi^{\ell}\left(\tilde{x}_{0}\right)\right) .
$$

Then, $\Phi^{k}\left(\tilde{x}_{0}\right)=\tilde{x}^{k}\left(\tilde{\tau}_{k}\right)$ and $\gamma^{k}\left(\tilde{x}_{0}\right)=\tilde{\tau}_{k}^{\prime}-\tilde{\tau}_{0}$ for the execution $\tilde{\chi}=(\tilde{\tau}, \tilde{q}, \tilde{x})$ with $\left(\tilde{q}_{0}, \tilde{x}_{0}\right) \in q_{0} \times W^{0}$. The functions $\Phi^{k}$ and $\gamma^{k}$ are continuous by construction. By the continuity of $\gamma^{N}$, there exists $\delta_{1}>0$ such that for all $\tilde{x}_{0}$ with $\left\|\tilde{x}_{0}-x_{0}\right\|<\delta_{1}$, we have $\left|\gamma^{N}\left(\tilde{x}_{0}\right)-\gamma^{N}\left(x_{0}\right)\right|<\epsilon$, or, in other words, $\left|\sum_{i=0}^{N}\left(\tilde{\tau}_{i}^{\prime}-\tilde{\tau}_{i}\right)-\sum_{i=0}^{N}\left(\tau_{i}^{\prime}-\tau_{i}\right)\right|<\epsilon$. By the continuity of $\Psi^{N}$ there exists $\delta_{2}>0$ such that for all $y \in W^{N}$ with $\left\|y-x^{N}\left(\tau_{N}\right)\right\|<\delta_{2}, \| \Psi^{N}(y)-$ $x^{N}\left(\tau_{N}^{\prime}\right) \|<\epsilon$. Hence, by the continuity of $\Phi^{N}$, there exists $\delta_{3}>0$ such that for all $\tilde{x}_{0} \in W^{0}$ with $\left\|\tilde{x}_{0}-x_{0}\right\|<$ $\delta_{3},\left\|\Phi^{N}\left(\tilde{x}_{0}\right)-x^{N}\left(\tau_{N}\right)\right\|<\delta_{2}$. Since $\Psi^{N}\left(\Phi^{N}\left(\tilde{x}_{0}\right)\right)=$ $\tilde{x}^{N}\left(\tilde{\tau}_{N}^{\prime}\right)$, we have $\left\|\tilde{x}^{N}\left(\tilde{\tau}_{N}^{\prime}\right)-x^{N}\left(\tau_{N}^{\prime}\right)\right\|<\epsilon$. The proof is completed by setting $\delta=\min \left(\delta_{1}, \delta_{3}\right)$.

Proof of Theorem 4.1: Consider an arbitrary state $\left(q_{0}, x_{0}\right) \in \Omega$ and let $\chi=(\tau, q, x) \in \mathcal{E}_{H}^{\infty}\left(q_{0}, x_{0}\right)$. Since $\Omega$ is invariant, $\left(q(i), x^{i}(t)\right) \in \Omega$ for all $i \in\langle\tau\rangle$ and $t \in I_{i}$. Since $\Omega$ is compact and $V$ is continuous, $V\left(q(i), x^{i}(t)\right)$ is bounded from below. Moreover, $V\left(q(i), x^{i}(t)\right)$ is a non-increasing function of $i \in\langle\tau\rangle$ and $t \in I_{i}$ (recall that $\tau$ is linearly ordered), therefore the limit $c=\lim _{t \rightarrow \mathcal{T}(\chi)} V\left(q(i), x^{i}(t)\right)$ exists.

Since $\Omega$ is bounded, $x$ is bounded, and therefore the $\omega$-limit set $S_{\chi}$ is nonempty by Lemma 4.1 . Since $\Omega$ is closed, $S_{\chi} \subset \Omega$. By definition, for any $(\hat{q}, \hat{x}) \in S_{\chi}$, there exists a sequence $\left\{\theta_{n}\right\}_{n=0}^{\infty}$ with $\theta_{n} \in I_{i_{n}}, i_{n} \in\langle\tau\rangle$ such that as $n \rightarrow$ $\infty, \theta_{n} \rightarrow \mathcal{T}(\chi)$ and $\left(q\left(i_{n}\right), x^{i_{n}}\left(\theta_{n}\right)\right) \rightarrow(\hat{q}, \hat{x})$. Moreover, $V(\hat{q}, \hat{x})=V\left(\lim _{n \rightarrow \infty}\left(q\left(i_{n}\right), x^{i_{n}}\left(\theta_{n}\right)\right)=\right.$ $\lim _{n \rightarrow \infty} V\left(q\left(i_{n}\right), x^{i_{n}}\left(\theta_{n}\right)\right)=c$, by continuity of $V$. Since $S_{\chi}$ is invariant (Lemma 4.1), it follows that $L_{f} V(\hat{q}, \hat{x})=0$ if $(\hat{q}, \hat{x}) \notin \operatorname{Out}_{H}$, and $V\left(\hat{q}^{\prime}, R(\hat{e}, \hat{x})\right)=$ $V(\hat{q}, \hat{x})$ if $(\hat{q}, \hat{x}) \in \mathrm{Out}_{H}$ and $\hat{e}=\left(\hat{q}, \hat{q}^{\prime}\right) \in E$. Therefore, $S_{\chi} \subset S_{1} \cup S_{2}$, which implies that $S_{\chi} \subset M$ since $S_{\chi}$ is invariant and $M$ is maximal. By Lemma 4.1, as $t \rightarrow \mathcal{T}(\chi)$, the execution $\chi$ approaches $S_{\chi}$, and hence $M$. 